Strahlenther Onkol 2012 .

[Suppl 3] ·188:356-356

DOI 10.1007/s00066-012-0212-2

Online publiziert: 10. August 2012

๑) Springer-Verlag Berlin Heidelberg 2012

U.M. Lütolf

Klinik für Radioonkologie, Universitätsspital Zürich

\title{
Bedeutung der Strahlentherapie und Onkologie für die Schweizer Radioonkologie
}

(Scientific Association of Swiss Radiation Oncology, gegründet 1996 in Bern) und die Mehrsprachigkeit der Schweiz legten dann den Fokus der Präsentationen auf die englische Sprache.

Was hat sich seit der Mitherausgeberschaft von Strahlentherapie und Onkologie im Jahre 1997 geändert? Die institutionalisierte Mitherausgeberschaft des jeweiligen Präsidenten der SASRO eröffnete der Schweizer Radioonkologie ein Forum zur Präsentation der Jahrestagungen und verhalf der jungen Gesellschaft unabhängig von der diagnostischen Radiologie zu einer wissenschaftlichen Plattform.

Obwohl Schinz bereits seit 1923 Mitherausgeber der Strahlentherapie war, finden sich viele seiner Publikationen in $\mathrm{On}$ cologia, der „Zeitschrift für Erforschung, Bekämpfung, Behandlung und Soziologie der Krebskrankheit“, dem wissenschaftliches Organ der „Schweizerischen Nationalliga für Krebsbekämpfung und Krebsforschung“, seit 1967 Oncology. Schinz wurde auch selbst deren Herausgeber.

Die Mitherausgeberschaft von Strahlentherapie und Onkologie durch die SASRO seit 1997 hat in der Schweiz die Einzelpersonen, die in den ersten 50 Jahren in der Strahlentherapie das Feld beherrschten, in den Hintergrund treten lassen. Mit der englischen Sprache, die nach und nach die deutschsprachigen Beiträge ersetzte, stieg auch deren Attraktivität. Die Zahl der pro Jahr an die Strahlentherapie eingereichten und akzeptierten Publikationen ist konstant geblieben. Das anfallende „Mehr“ an wissenschaftlichen $\mathrm{Pu}$ blikationen aus der Schweiz floss dem „Red Journal“ (Int J Rad Oncol Biol Phys) und dem „Green Journal“ (Radiother Oncol), also der „Konkurrenz“ zu. Allerdings sind aus der welschen Schweiz weiterhin nur ca. 20\% der Beiträge der letzten Jahre, die radioonkologisch, fachspezifisch veröffentlicht wurden, in der Strahlentherapie zu finden. Aus der Deutschen Schweiz sind dies nach wie vor mehr als 50\%.

Aus den "goldenen Zeiten“ der Wissenschaftswelt mit einer identitätsstiftenden Zeitschrift für die deutschsprachigen Strahlentherapeuten ist ein international geachtetes Journal geworden, das wohl mit sicherer Hand aus Erlangen geführt wird, der Nachbarin Schweiz mit ihren Alpenübergängen aber ein Tor in eine neue Zeit zu öffnen wusste.

\section{Korrespondenzadresse}

Prof. Dr. U.M. Lütolf

Klinik für Radioonkologie,

Universitätsspital Zürich

Zürich

Schweiz

Urs.Luetolf@usz.ch 